\title{
Discussion on Application of Pneumatic Devices in Printing Machinery
}

\author{
Huijie Zhao ${ }^{1}$, Guangping Wang ${ }^{2}$ \\ ${ }^{1}$ Shijiazhuang Staff and Workers University, Shijiazhuang, 050000, China \\ ${ }^{2}$ Hebei Economy and Management School, Shijiazhuang, 050000m China
}

Keywords: Pneumatic device; Printing machinery; Application principle

\begin{abstract}
With the development of society and progress in science and technology, the social competition has been fiercer and fiercer, printing factories also face more challenges and developments, and more and more printing factories hope to reduce the waste rate of printing products reasonably, so as to continuously improve the printing quality and increase printing speed, thus increasing the enterprise competitiveness and ensuring the very advantageous status of enterprises. Therefore, $t$ is required to continuously research new technologies, and improve the economic and social benefits of enterprises. The continuous application of pneumatic devices in printing can effectively improve the product precision, stability and production efficiency, and this is also the first objective for manufacturing of printing factories. This paper mainly researches the application of pneumatic devices in the printing machinery.
\end{abstract}

\section{Introduction}

Currently, the continuous development of science and technology, and the continuous research and deepening of electric elements and pneumatic devices provide guarantee and future development direction for printing machinery factories to increase the development of automation technology of printing machines. According to the specific condition of the domestic printing industry in the manufacturing of printing machinery, the technology of applying pneumatic devices in the printing machinery is has not been quite mature, and many users do not know how to use the pneumatic equipment and maintenance equipment, which influences the use effect of pneumatic device in the mechanical printing. However, in order to improve the application of pneumatic device in the printing enterprises and improve the function of the pneumatic devices, it is required to analyze the application of pneumatic devices in printing machinery in combination with the actual condition .

\section{Analysis on basic information of pneumatic devices in printing machinery}

At present, fundamentally, the printing machinery used by the current printing factories is the collection of binding machinery, printing machine, layout machine and various machinery and equipment as well as the corresponding auxiliary equipment. The essential function of such equipment is to use a certain technology to shift the ink in the printed tests to the printed material, and use it repeatedly and cyclically, so as the achieve the objective of mass printing. Domestically, the generally printing machinery and pneumatic devices are exported, like the pneumatic equipment produced by Heidelberg, Komori and Mitsubishi, which is widely applied in the printing machinery, with quite a broad application prospect. Pneumatic device is generally suitable for the automatic ink washing structure, water and ink transmission structure, disengaging and engaging impression structure, lock-up structure and water and ink dipping structure of the printing machinery, and such device has also been gradually used domestically, which can effectively ensure the printing efficiency and quality, so as to achieve the development and progress of printing industry and accelerate the development of national economy ${ }^{[1]}$. 


\section{Main problems of application of pneumatic device in printing machinery}

According to the composition structure, the pneumatic device generally consists of filter pressure relief valve, air compressor, cylinder and solenoid valve, which can provide certain guarantee and basis for the pneumatic equipment, and the following is the analysis on key problems of the four kinds of equipment.

\section{Air compressor}

Air compressor actually is the essential part of the whole printing pneumatic device, playing quite a key role for the equipment. In terms of the printing machinery, most of the mechanical energies in the pneumatic equipment system can be converted into the corresponding air pressure with air compressor, and the fundamental characteristic of such air compressor is that the mechanical energy can be converted to emit the gas mass for the whole purification system. Basically, in the work process of printing machinery, the atmospheric pressure parameters and air compressor that can be used should be maintained between 6 bar and 8 bar, and the parameter indicators of the air compressor displacement can be determined based on the maximum air volume value of the air compressor within the specified value. It is worth noting that when using the air compressor, we should comply with the use specifications of air compressor and user instructions for corresponding quantitative and timing drainage, so as to maximally prevent sewage flowing into the pneumatic equipment and ensure the normal use and running of the pneumatic equipment ${ }^{[2]}$.

\section{Filter pressure relief valve}

In the development and support of the current technology level, the filter pressure relief valve equipment generally consists of the pressure relief valve equipment and filter, which is the auxiliary equipment that cannot be obtained in the process of using pneumatic equipment. In the actual process of use, it cooperates with the air compressor so as to provide certain auxiliary technology for the printing machinery equipment. In this paper, the problems of pneumatic equipment in the process of using printing machinery are analyzed from the aspect of how to use pressure relief equipment and filter.

Filter

In the pneumatic equipment, filter plays quite an important role, which can separate the solid grain structure in the compressed air and the relatively large impurity and oil in the condensation water structure. From the aspect of using pneumatic equipment in the printing machinery, it is required to concern the following problems when using the filter: first, when using filter in the printing machinery, it is required to always keep the filter placed vertically and it should be put down. Second, when using the filter, it is required to pay attention to the daily maintenance and management in use, clan the filter regularly, and it is possible to well clear away the oil, condensate water and solid impurity, so as to ensure that the substances remaining in the filter will not exceed the actual requirements of the water fender in case of maximum influence on the actual performance of filter. In addition, when using the filter, we can also see such a phenomenon that there is a air leakage usually in the filter, and sometimes, the sealing of filter will be greatly influenced, and the essential reason for such problem is the installation problem. Overall, when installing the filter, it is required to strictly comply with the actual arrow of the air flow transmission direction, so as to reasonably find out the most appropriate installation direction.

Valve relief valve equipment

When using pneumatic equipment in the printing machinery, it is required to reasonably install a pressure relief valve to provide the corresponding air pressure regulation capacity, it is possible to appropriately accept the gas brought by the air compressor and conduct reasonable and appropriate processing and regulation, so as to ensure that the air pressure output can meet the actual demand and parameters of the printing machinery. Basically like the air compressor, the air pressure in the pressure relief valve should be maintained at 6-8bar to avoid cylinder damage in case of great pressure. If the air pressure is small, the operation efficiency of the printing machine might be 
relatively low, in case of air leakage to the pressure relief valve in the use of pneumatic equipment, the main work is to find out the source in the sealing washer, reasonably stop the printing machinery, and timely replace and repair the parts, so as to ensure that the printing machinery can maintain normal operation ${ }^{[3]}$.

\section{Solenoid valve}

Generally, during the running of pneumatic device of the printing machinery, the solenoid valve can control the movement of all pneumatic equipment and cylinder equipment and the movement direction of air flow. When the piston rod in the cylinder equipment can run outside, the air channel will be connected to the right position of the solenoid valve, so as so to ensure that the air can enter from the rear part of the cylinder and be exhausted from the front part, thus the piston can move outside better. When the cylinder piston rod should run inside, the air channel with be connected to the left position of the solenoid equipment, so that the air outlet position is opposite to the inlet position, so as to ensure the inward running of the piston. In the actual running of printing machine, we might find that the cylinder is out of function or has wrong movement. When judging the reasons, we should comply with the following: suspend the work of printing machine, plug out the air inlet pipe device, run the solenoid valve manually and observe whether there will be air generated in the air inlet pipe. In case of air, it indicates that it is caused by the fault, and it is required to replace or repair the equipment, but if there is no air, it indicates that it is caused by the fault of solenoid valve equipment, and it is required to directly replace and restart the pneumatic equipment, so as to ensure that the machinery can maintain the normal running.

\section{Cylinder}

As the essential executive device of the pneumatic equipment and also the core and foundation of the pneumatic equipment, the cylinder can provide the corresponding compressed air for the system transmission process and reasonably convert it into certain mechanical energy, so as to ensure the normal running of the cylinder. In case of cylinder problem during the running of printing machinery, we should use the real-time detection meter to detect the pressure parameter, if there are still some pressures, it is required to further detect the solenoid equipment, and such detection method is basically the same with the solenoid valve detection method ${ }^{[4]}$.

\section{Application of pneumatic device in printing machinery}

At present, pneumatic technology has been gradually applied in a lot of printing machinery like roll-fed offset printing press, sheet fed offset printing press and intaglio printing press, and in the process of work of printing machinery, some pneumatic equipment is required to achieve the overall running of the equipment. During the delivery and separation, sheet fed offset printing press should need the paper delivery nozzle, paper separation nozzle and paper separation blowtorch. The paper is transmitted to the paper transmission panel in the form of vacuum paper transmission, pneumatic control is used to achieve the disengaging and engaging impression of the printing equipment, and vacuum absorption is used for paper withdrawal deceleration ; when the roll-fed paper rotary press is used, the pneumatic technology is used to control the tension, disengaging and engaging pressure of the printing machine and control the position of mechanism in the paper feeding device; when intaglio printing press is used, pneumatic system is used to reasonable control the drive and separation, cut-off knife action, cut-off knife frame falling, engaging and disengaging between the pressing roller and forme and the position of roller in the paper feeding and withdrawal. There are mainly two methods of using pneumatic device in the printing machinery, one is to use appropriate air to ensure the requirement and work in the process of printing, and the work is mainly completed by the positive and negative pressures of the gas, which is generally suitable for the paper delivery nozzle, vacuum paper transmission device and paper separation nozzle, paper blowtorch as well as the paper deceleration equipment. The blowing and inhalation are mainly completed with the pore or nozzle, such work only needs the air source, and the specified air blowing and inhalation can be 
conducted under a certain pressure, or the pore can be connected to the air for a certain position, and such work is relatively easy to be completed. Another work mode is actually to use different solenoids to control the cylinder piston to control the operation, reasonably control the engaging and disengaging equipment, ink and water dipping rollers etc.

\section{Pneumatic control in printing disengaging and engaging impression device}

No matter the type of the printing machinery is, during printing, the impression mechanism in the printing equipment should mutually contact with the lock-up mechanism, and bear a certain printing pressure, and the printing equipment of such printing condition is called throwing-in. Once in case of paper transmission fault or paper transmission system top or preparations are made, the impression mechanism and the lock-up mechanism can be disintegrated timely to remove the printing pressure, and the printing equipment of such condition belongs to throwing-off. The throwing-in and throwing-off are mainly achieved by changing the central distance of the roller, and electric appliance control is used to achieve the execution of throwing-in and throwing-off of the printing device. In the earliest period, the PZ4880A folio four-color offset press and Beiren J2108A folio single-color offset press invented by the Chinese completed the driving of engaging and disengaging reasonably by controlling the electric magnetic. J2108A folio single-color offset press is mainly to drive the throwing-in cam throwing-off cam on the impression roller and eccentric shaft of four-rod equipment for swaying back and forth, so as to reasonable achieve the engaging and disengaging by controlling the electric magnetic. The electric magnetic is regarded as the executive equipment of the engaging and disengaging impression to reasonably control the relatively complicated engaging and disengaging position. The use of pneumatic control technology to achieve the change of engaging and disengaging pressure is mainly to use the movement condition of piston to reasonably completely the swaying and running of four-rod equipment and achieve the engaging and disengaging impression of the printing machinery by changing the connecting rod, such reasonable use of electric magnetic to complete the movement of connecting rod is not so reliable, and it is easy to cause shock and vibration. Compared with the traditional method, the essential advantage of using pneumatic control technology to ensure the operation of engaging and disengaging impression device of the pneumatic control technology is that it can simplify the mechanical structure in a certain extent, reduce the use efficiency of parts and components in the mechanical running, without throwing-in and throwing-off cams, which more ensures the running reliability and improves the equipment control accuracy. The pneumatic control equipment itself has the advantages of being accurate, simple and stable, so generally pneumatic control system is used to achieve the engaging and disengaging impression control of the printing machine.

\section{Pneumatic control system}

When selecting cylinder, first it is required to reasonably select the installation form. During selection, it is not only required to meet the straight movement of piston, but also the arc swaying, therefore, pin-type cylinder is relatively suitable. It is required to select the working force according to the cylinder fixing tension and thrust needed in the specific work condition and reasonably determine and calculate the main cylinder parameters, if the relatively standard cylinder is used, it is required to calculate the cylinder diameter reasonably for reasonable calculation, and it is also required to maintain a certain allowance, promote the running of expansion mechanism, so as to reduce the cylinder size, and the length of stroke is selected by adding $10-20 \mathrm{mmd}$ allowance in the stroke.

\section{Conclusion}

In a word, with the continuous construction and promotion of society, economy and urbanization process, the market competition has been fiercer and fiercer, bringing unprecedented challenges and opportunities to all enterprises, and the printing enterprises also have been gradually shocked. How to continuously improve the enterprise competitiveness in the overall context of fierce market 
competition is an issue that the enterprises quite emphasize, applying pneumatic device in the printing machinery can effectively improve the production quality and efficiency, and has been gradually concerned by all circles of the society, so it is quite important to strengthen the research.

\section{References}

[1] Cheng Sheng, Exploration on Application of Pneumatic Device in Printing Machinery, Chinese High Technology Enterprises, 2012(4):71-72.

[2] Shi Lihua, Sun Changli, Xu Yongxin et al., Application of Pneumatic Device in Printing Machinery, Printing Field, 2011(3):61-62.

[3] Yuan Zhongzeng, Application of Automatic Control Technology in Printing Machinery, Technology Wind, 2013(14):84-84.

[4] Pan Jie, $\mathrm{Xu}$ Zhongliu, Current Status of Export of Chinese Printing Machinery and Countermeasures for Expansion of Export, Printing Field, 2010(11):30-34.

[5] Development Planning of Printing Machinery Industry in the "12th Five-year Plan” Period of China issued, to achieve gross industrial output of printing machinery industry RMB 40 billion at the end of "12th Five-year Plan” period, Journal of Beijing Institute of Graphic Communication, 2011,19(4):41-41. 